\title{
ENQUÊTE SUR LA RÉPARTITION DES VIRUS DES SALMONIDÉS DANS LES SALMONICULTURES DE LA RÉGION AQUITAINE ET DES RÉGIONS LIMITROPHES
}

\author{
Ph. NOUgayrede
}

\author{
Laboratoire Vétérinaire Départemental des Landes - LVD 40 - Directeur : A. VUILLAUME \\ 120, avenue Pasteur - 40000 MONT-DE-MARSAN
}

Reçu le 17 octobre 1988

Accepté le 23 décembre 1988

Received 17 October, 1988

Accepted 23 December, 1988

\section{RÉSUMÉ}

Dans le Sud-Ouest de la France, 599 prélèvements de truites AEC (Salmo gairdneri) (71\%), de truites fario (Salmo trutta) (14\%), d'ombles de fontaine (Salvelinus fontinalis) (1\%), de saumons atlantiques (Salmo salar) (2\%), et d'espèces diverses (12\%), ont été analysés pendant une période de 2 ans.

Le virus de la Septicémie Hémorragique Virale (S.H.V.) a été trouvé sur $6 \%$ des prélèvements répartis sur 15 piscicultures, et le virus de la Nécrose Pancréatique Infectieuse (N.P.I.) sur $42 \%$ des prélèvements. Le virus de la N.P.I. a été trouvé sur les 55 piscicultures régulièrement contrôlées en Aquitaine.

\section{INVESTIGATION ABOUT THE DISTRIBUTION OF SALMONID VIRUSES FROM THE PISCICULTURES IN "AQUITAINE" AND AROUNDING COUNTRIES}

\section{SUMMARY}

During a period of two years, 599 samples of rainbow trout (S. gairdneri) $(71 \%)$, brown trout (Salmo trutta) (14\%), brook trout (Salvelinus fontinalis) (1\%), atlantic salmon (Salmo salar) (2\%), and a various species $(12 \%)$, were analysed. The viral haemorrhagic Septicaemia (V.H.S.) virus is present in about $6 \%$ of samples from 15 piscicultures and infectious pancreatic necrosis virus is present in about $42 \%$ of samples from the 55 piscicultures regulary inspected in Aquitaine.

\section{INTRODUCTION}

Cette note a pour but de présenter une enquête sur la répartition des virus des salmonidés dans les salmonicultures de la Région Aquitaine à partir des résultats obtenus dans un laboratoire de diagnostic des maladies virales des poissons récemment créé dans cette région oủ l'élevage des salmonidés est très important ( 8.000 tonnes en 1985, soit $1 / 3$ de la production française).

Le diagnostic des viroses est fondamental en Ichtyopathologie d'une part, parce que les symptômes et les lésions observés sur les poissons sont rarement pathognomoniques d'une infection, d'autre part, parce que l'absence de diagnostic virologique peut entraîner une erreur de diagnostic avec son cortège de traitements coûteux et inutiles.

L'impact économique des viroses, difficile à chiffrer avec exactitude, se fait principalement sentir dans les conditions de l'élevage intensif des salmonidés (de KINKELIN, 1985) qui s'est développé, principalement dans le département des Landes, durant ces 10 dernières années. La connaissance de la répartition des virus des salmonidés est essentielle dans une région pour l'application de mesures sanitaires efficaces qui visent à protéger les piscicultures indemmes, à contrôler les repeuplements des rivières et à assainir les piscicultures et les rivières contaminées.

\section{MATÉRIEL ET MÉTHODE}

\section{Origine des prélèvements}

Les observations présentées dans cette note portent sur les analyses effectuées entre le $1^{\text {er }}$ janvier 1986 et le 31 décembre 1987.

599 prélèvements ont été examinés en provenance d'une dizaine de départements français (région Aquitaine : Pyrénées-Atlantiques, Landes, Gironde, Lot et Garonne et Dordogne, quelques départements limitrophes: Hautes-Pyrénées, Gers, Charente, Aveyron, Tarn) et d'un pays européen voisin, l'Espagne. 
Les prélèvements ont été effectués, soit lors d'un contrôle sanitaire dans le cadre du Groupement de Défense Sanitaire Aquacole Aquitain (G.D.S. Aquacole), ou de l'inscription des piscicultures au Contrôle Officiel, soit à l'occasion d'un problème pathologique nécessitant une analyse virologique pour confirmer ou infirmer le diagnostic.

Le Laboratoire de virologie a reçu 599 prélèvements d'organes provenant d'autopsies effectuées, soit sur la pisciculture dans la camionnette laboratoire du G.D.S. aquacole, soit dans la salle d'autopsie du Laboratoire Départemental des Services Vétérinaires des Landes.

Le Tableau I détaille l'origine géographique des prélèvements et les diverses espèces analysées au Laboratoire.

Tableau I: Localisation géographique, espèces de poisson et nombre de prékèvements analysés au Laboratoire pendant une période de 2 ans (du 1/1/86 au 31/12/87).

Table 1: Geographical localization, tish species and number of samples analysed in the Laboratory for a period of two years $(1 / 1 / 86-31 / 12 / 87)$.

\begin{tabular}{|c|c|c|c|c|c|c|c|c|c|c|c|c|c|c|c|}
\hline Départements & 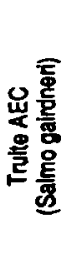 & 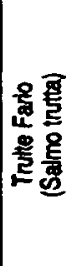 & 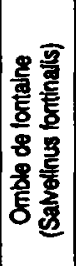 & 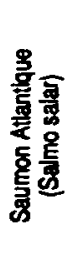 & 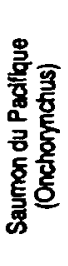 & 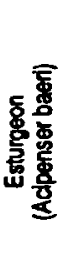 & 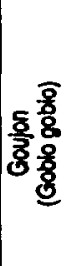 & 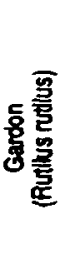 & 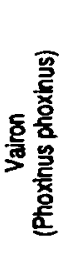 & 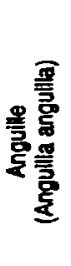 & 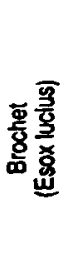 & 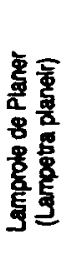 & 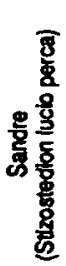 & 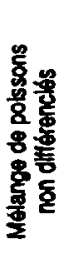 & 령 \\
\hline DORDOGNE & 2 & 0 & 0 & 3 & 0 & 0 & 0 & 0 & 0 & 0 & 0 & 0 & 0 & 0 & 5 \\
\hline GIRONDE & 31 & 5 & $\mathbf{0}$ & 0 & 0 & 0 & 0 & 0 & 0 & 0 & 0 & $\mathbf{0}$ & 1 & D & 37 \\
\hline LANDES & 200 & 39 & 2 & 0 & 4 & 23 & 5 & 2 & 4 & 2 & 2 & 1 & 0 & 9 & 295 \\
\hline LOT ET GAFONNE & 17 & 3 & 2 & o & 0 & 0 & 0 & 1 & 1 & 0 & 0 & 0 & 0 & 0 & 24 \\
\hline PYRENES ATL. & 102 & 28 & 2 & 5 & 0 & o & 0 & $\mathbf{0}$ & 1 & 0 & $\mathbf{0}$ & $\mathbf{0}$ & 0 & 0 & 138 \\
\hline HAUTES PYRÉNÉES & 15 & 2 & 1 & 0 & 0 & 11 & 0 & $\mathbf{0}$ & 0 & 0 & 0 & o & 0 & 0 & 29 \\
\hline TARN & 1 & 1 & 0 & 3 & 0 & 0 & 0 & 0 & 0 & 0 & 0 & $\mathbf{0}$ & 0 & 0 & 5 \\
\hline AVEYRON & 0 & 1 & 0 & 0 & 0 & 0 & 0 & 0 & 0 & 0 & 0 & 0 & 0 & 0 & 1 \\
\hline GERS & 2 & 0 & 0 & 0 & 0 & 0 & 0 & 0 & 0 & 0 & 0 & 0 & 0 & 0 & 2 \\
\hline CHARENTE & 6 & 3 & 1 & 0 & 2 & 0 & 0 & 0 & 0 & 0 & 0 & 0 & 0 & 0 & 12 \\
\hline AUTRES DEPART. & 3 & 1 & 0 & 0 & 0 & 0 & 0 & 0 & 0 & 0 & 0 & o & 0 & 0 & 4 \\
\hline PAYS AUTRES & 45 & 1 & 0 & 0 & 0 & 0 & $\mathbf{0}$ & 0 & 0 & 0 & 0 & 0 & 0 & 1 & 47 \\
\hline TOTAL & 126 & 84 & 8 & 11 & 6 & 34 & 5 & 3 & 6 & 2 & 2 & 1 & 1 & 10 & 599 \\
\hline
\end{tabular}

Dans la plupart des cas, les recherches ont été effectuées sur du poisson d'élevage : truites Arc-en-Ciel (Salmo gairdneri) essentiellement ( $71 \%$ des cas), mais aussi des truites fario (Salmo trutta fario), des ombles de fontaine (Salvelinus fontinalis), des saumons atlantiques (Salmo salar) et des esturgeons (Acipenser baeri). 25 prélèvements provenaient de poissons et agnathes sauvages (truites fario, vairons, gardons, anguilles, lamproies de Planer, goujons, brochets) prélevés lors de péches électriques destinées à contröler l'environnement des piscicultures infectées par le virus de la Septicémie Hémorragique Virale (S.H.V.).

\section{Techniques utilisees}

Les analyses ont été effectuées selon les techniques décrites par de KINKELIN et al. (1985), KEVIN, (1985). Sur un échantillon de poissons (5 à 10 sujets en général), les reins antérieurs, la rate et le cerveau sont prélevés. Ils sont broyés, dilués $(1 / 10,1 / 100,1 / 1000)$ et ensemencés sur des cellules Rainbow trout Gonad RTG 2 (WOLF et QUIMBY, 1962) et/ou Epithelium papulosum cyprini E.P.C. (FIJAN et al., 1983) cultivées sur des microplaques (boites multiples à 24 cupules Nunclon delta) dans un milieu BHK 21 additionné de Tryptose phosphate broth $(10 \%)$ et de sérum de veau fœetal $(2 \%)$. Les microplaques sont incubées à $15^{\circ} \mathrm{C}$. Des lectures pour recherche de l'effet cytopathique caractéristique des virus sont effectuées quotidiennement pendant 10 jours. Trois passages sont effectués avant de rendre un résultat négatif.

Le sérotypage des souches, quand il a eu lieu, a été effectué par séroneutralisation au Laboratoire d'Ichtyopathologie de l'INRA. 


\section{Virus recherchés}

Les virus de la Nécrose Pancréatique Infectieuse (N.P.I.) (COHEN et al., 1973; DORSON et al., 1978), et les rhabdovirus des salmonidés (Virus de la Septicémie Hémorragique Virale (S.H.V.) (ZWILLENBERG et al., 1965), et Virus de la Nécrose Hématopoïétique Infectieuse (N.H.I.) (Mc ALLISTER et al., 1974 ; DARLINGTON et al., 1974) ont été systématiquement recherchés même à partir de poissons porteurs passifs ou actifs avec infection inapparente dans le cas d'esturgeons et poissons blancs par exemple. l'élevage.

En effet, nous avons alors recherché un portage des virus des salmonidés pouvant nuire à

\section{RESULTATS-DISCUSSIONS}

Les résultats globaux sont donnés dans le Tableau II. On constate que sur 599 analyses pour lesquelles les rhabdovirus et les virus de la NPI ont été recherchés, 249 prélèvements se sont révélés positifs en NPI $(42 \%)$ et 38 en rhabdovirus $(7 \%)$. C'est à partir des truites AEC que les résultats sont les plus intéressants en raison du nombre d'analyses. Sur la moitié des analyses, nous avons trouvé le virus de la NPI (53\%) et sur environ $10 \%$ un rhabdovirus. Dans un seul cas seulement, nous avons trouvé conjointement le virus de la NPI et un rhabdovirus.

Tableau II : Résultat des examens virologiques effectués au Laboratoire pendant une période de 2 ans (du 1/1/86 au 31/12/87).

Table II : Results of virologic analyses examined in the Laboratory for a period of two years (1/1/86 - 31/12/87).

\begin{tabular}{|c|c|c|c|c|c|}
\hline & \multirow[t]{2}{*}{$\begin{array}{l}\text { Nombre } \\
\text { d'analyses }\end{array}$} & \multicolumn{2}{|c|}{$\begin{array}{c}\text { Présence d'un virus de la Nécrose Pancréatique } \\
\text { Infectieuse }\end{array}$} & \multicolumn{2}{|c|}{ Prêsence d'un rhabdovirus } \\
\hline & & $\begin{array}{l}\text { Nore analyses } \\
\text { positives }\end{array}$ & \% & $\begin{array}{l}\text { Nbre analyses } \\
\text { positives }\end{array}$ & 8 \\
\hline $\begin{array}{l}\text { Truite Arc en Ciel } \\
\text { (Salmo gairdneri) }\end{array}$ & 426 & 224 & $53 \%$ & 37 & 92 \\
\hline $\begin{array}{l}\text { Truite fario } \\
\text { (Salmo trutta) }\end{array}$ & 84 & 20 & $24 \%$ & 0 & \\
\hline $\begin{array}{l}\text { Omble de fontaine } \\
\text { (Salvelinus fontina- } \\
\underline{\text { lis })}\end{array}$ & 8 & 3 & $38 \%$ & 0 & \\
\hline $\begin{array}{l}\text { Saumon at lantique } \\
\text { (Salmo salar) }\end{array}$ & 11 & 0 & & 1 & $9 \%$ \\
\hline $\begin{array}{l}\text { Saumon du Pacifique } \\
\text { (Onchorynchus) }\end{array}$ & 6 & 0 & & 0 & \\
\hline $\begin{array}{l}\text { Esturgeon } \\
\text { (Acipenser baeri) }\end{array}$ & 34 & 1 & $3 x$ & 0 & \\
\hline Vairon & 6 & $\mathbf{1}$ & $17 \%$ & 0 & \\
\hline $\begin{array}{l}\text { Goujon, gardon, sandr } \\
\text { anguille, brochet, } \\
\text { lamproie }\end{array}$ & 24 & 0 & & 0 & \\
\hline TOTAL & 599 & 249 & $42 x$ & 38 & $7 \%$ \\
\hline
\end{tabular}

Dès le premier passage, il a été possibłe de conclure à la présence d'un rhabdovirus dans tous les cas ; par contre, dans environ $10 \%$, l'effet cytopathogène caractéristique d'un virus de la NPI n'a été détecté qu'au $2^{e}$ passage sur culture cellulaire. Les résultats sont donnés négatifs, quand après 3 passages sur culture cellulaire, il n'y a aucun effet cytopathogène. 
Dans un cas, nous avons isolé un rhabdovirus sur des alevins de saumons atlantiques. Bien que le nombre d'analyses soit plus réduit, nous pouvons constater que le virus de la NPI est trouvé assez souvent sur des truites fario et des saumons de fontaine. Exceptionnellement, il a été mis en évidence sur des vairons (1 cas) et sur des esturgeons (1 cas). Les typages concernant les rhabdovirus isolés pendant cette période ont été réalisés au Laboratoire d'lchtyopathologie de l'INRAGrignon.

A chaque fois un virus de la Septicémie Hémorragique Virale a été mis en évidence. Dans un cas apparu dans les Landes, ce virus appartenait au sérotype 23-75 (type 3) (LE BERRE et al., 1977).

La souche isolée sur les saumons atlantiques a été neutralisée par l'anticorps monoclonal C 10 (anti groupe SHV), mais non par le sérum anti SHV 1 et le sérum anti NHI. II s'agissait donc d'une souche de virus SHV de types autres que le 1 (VESTERGAARD-JORGENSEN, 1972; de KINKELIN et LE BERRE, 1977).

Il faut noter que ces résultats obtenus à partir de prélèvements faits tout le long de l'année (mois d'été compris) sur différents lots ne permettent pas de conclure sur l'incidence des viroses dans une région. Pour cela, il faut raisonner en site d'élevage (pisciculture). Au cours des deux années d'examen, le virus de la NPI a été trouvé sur la totalité des 55 piscicultures soumises à contrôles réguliers dans les départements d'Aquitaine (Pyrénées-Atlantiques, Dordogne, Landes, Gironde, Lot-et-Garonne). Il a été mis en évidence sur des alevins présentant des signes cliniques évoquant la Nécrose Pancréatique, mais aussi sur des truites reproductrices avec localisation unique dans le tissu cérébral confirmant une observation déjà faite par DORSON (1982).

En ce qui concerne la recherche des rhabdovirus, les 38 analyses positives ont concerné 16 sites. 5 sites dans les Landes, 6 sites dans les Pyrénées-Atlantiques, 2 sites dans le Tarn, 1 site en Lot-et-Garonne, 1 site dans les Hautes-Pyrénées, et 1 site hors de France.

Dans les Pyrénées-Atlantiques, les 6 sites concernés sont tous situés sur la même rivière. Dans les Landes et en Lot-et-Garonne, l'abattage des animaux et un vide sanitaire ont permis l'élimination de la maladie, sauf sur un bassin hydrographique où de nouveau un rhabdovirus a été isolé en 1988. Les recherches virales faites sur le poisson sauvage, et notamment les truites présentes dans le cours d'eau en aval des piscicultures se sont toujours révélées négatives.

\section{CONCLUSION}

L'enquête sur les deux viroses majeures des salmonidés dans les piscicultures de la Région Aquitaine et des environs a permis de mettre en évidence, d'une part l'omniprésence du virus de la Nécrose Pancréatique Infectieuse, d'autre part, la présence de foyers très localisés de Septicémie Hémorragique Virale.

Aucun virus de la Nécrose Hématopoïétique Infectieuse, n'a été mis en évidence dans le cheptel du Sud-Ouest. L'application des règles sanitaires élémentaires, notamment le contrôle sérieux des mouvements de poissons, avec l'appui du G.D.S. Aquacole devrait permettre à cette région de rester indemne.

Dans la plupart des cas de SHV, l'abattage des animaux suivi d'un nettoyage, d'une désinfection et d'un vide sanitaire ont montré leur efficacité.

En ce qui concerne la NPI, le portage du virus par de nombreuses espèces autres que les salmonidés (DORSON, 1982) est confirmé au cours de cette enquête notamment pour le vairon.

Quant à l'esturgeon (Acipenser baeri), c'est la première fois que le virus de la NPI est isolé (CHENE, 1989). D'autres études sont en cours en collaboration avec l'équipe du CEMAGREF de Bordeaux. Il est important de noter que le portage du virus NPI par la quasi totalité des alevins de salmonidés produits dans la Région Aquitaine et par de nombreux poissons, autres que des salmonidés va rendre difficile l'éradication de cette virose dans les années à venir.

\section{REMERCIEMENTS}

Nos remerciements vont tout d'abord à $P$. de KINKELIN, Monique BEARZOTTI et toute l'équipe du Laboratoire d'Ichtyopathologie de l'INRA qui nous ont fait découvrir le diagnostic des viroses des poissons et qui nous ont fourni tous les réactifs nécessaires; aux différents membres du Groupement de Défense Sanitaire Aquacole d'Aquitaine pour leur aide et à Solange RIZZETTO pour la frappe du document. 


\section{BIBLIOGRAPHIE}

CHENE P. La pathologie de l'esturgeon, Thèse Doctorat Vet. Lyon, 1989 à paraître.

COHEN J., POINSARD A., SCHERRER R., 1973, Physico-chemical properties and morphological features of infections pancreatic necrosis virus. J. Gen. Virol., 21, 485-498.

DARLINGTON R.W., TRAFFORD R., WOLF K., 1974, Fish rhabdoviruses: morphology and ultra structure of north american salmonid isolates Arch. Geo. Virus forsch, 39, 257-264.

DORSON M., CASTRIC J., TORCHY C., 1978, Infectious pancreatic necrosis virus of salmonids: biological and antigenic features of a pathogenic strain and of a non pathogenic variant selected in RTG 2 cells. J. Fish Disease, 1, 309-320.

DORSON M., 1982, Nécrose Pancréatique Infectieuse des Salmonidés: Etat des connaissances concernant les virus et les possibilités de lutte contre la maladie, Bull. Fr. Piscic., 285, 195-209.

FIJAN N.. SULIMANOVIC D., BEARZOTTI M., MUZINIC D., ZWILLENBERG L.O., CHILMONCZYKS., VAUTHEROT J.F., de KINKELIN P., 1983, Somes properties of the Epithelioma papulosum cyprini (EPC) cell line from carp Cyprinus Carpio. Ann. Virol (Inst Pasteur), 134 E, 207-220.

KEVIN H. Amos, Editor, 1985, Procedures for the detection and identification of certain fish pathogens, 3d Ed. Fish Health Section, American Fischeries Society. Corvallis, Oregon.

KINKELIN P. (de), et LE BERRE M., 1977, Isolement d'un rhabdovirus pathogène de la truite Fario (Salmo trutta) C.R. Acad. Sc. Paris, 284 (Série D), 101-104.

KINKELIN P. (de), MICHEL C., GHITTINO P., 1985, Précis de Pathologie des poissons, INRA/OIE, Paris $348 \mathrm{p}$.

LE BERRE M., de KINKELIN P., METZGER A., 1977. Identification sérologique des rhabdovirus de salmonidés. Bull. Off. int. Epiz. 87, 391-393.

MC ALLISTER P.E., FRYER J.L., PILCHER K.S., 1974, Further characterization of infectious hematopoietic necrosis virus of salmonid fish (Oregon strain). Arch. ges. virus forsh., 44, 270-279.

VESTERGAARD-JORGENSEN P.E., 1972, Egtved virus: Antigenic variation in 76 virus isolates examined in neutralization tests and by means of the fluorescent antibody technique. In symposium of the zoological society of London, Academic Press, № 30, 333-339.

WOLF K. et QUIMBY M.C., 1962, Established eurythermic line of fish cells in vitro, Science, 135, 1065-1066.

ZWILLENBERG L.O., JENSEN M., ZWILLENBERG H., 1965. Electron microscopy of the virus of viral hemorrhagic septicemia of rainbow trout. Arch. Ges. Virus forsch, 17, 1-19. 